Townsend was elected to the new Wykeham chair in 1900, together with a fellowship at New College, R. B. Clifton being the existing professor of experimental philosophy. Until means for a laboratory were found, Townsend worked in the Observatory and later in the University Museum. It was not until 1910 that, with the help of the Draper's Company, the Electrical Laboratory was built, a stone building with walls $3 \mathrm{ft}$. thick, which was specially designed for electric and magnetic research.

Townsend's early work included a study of solar disturbances, magnetization of liquids, and the effects of secondary X-rays; but very soon he turned his attention to the motion of swarms of electrons in gases, in particular their motion in electric and magnetic fields. He recognized very early that drifting electron swarms could be treated as a flow of a gas passing through another stationary gas. Later he developed the concept of a 'hot electron gas' which is driven by the electric field through the cold parent neutral gas, the average energy of the electrons being orders of magnitude higher than that of the neutral molecules. This is because the light electrons are able to transfer only a small fraction of their energy to the heavy molecules in collisions. He also showed that the ionic charge in a gas is the same as in a liquid electrolyte.

From his numerous outstanding contributions one recalls the first measurements of the drift velocity of electrons (with Lattey) and its more accurate determination by deflecting the electron swarm by $a_{0}$ transverse magnetic field, of diffusion and random velocity of electrons in gases, and of the average energy losses which the electrons suffer in collisions with gas molecules (with Pidduck). To him also must be given the credit for the discovery of the dependence of the mean free path of electrons on the mean electron energy of swarms which he found in argon, now known as the Ramsauer-Townsend effect. He will be remembered for his fundamental work on the multiplication of charges by electron collisions and the dependence of the (Townsend) ionization coefficients on the electric field. He supported the first quantitative research by Kirkby on electro-chemistry of ionized gases. He showed that electrons and sometimes ions ionize gases, and that positive ions release secondary electrons from the negative electrode, thus increasing the current flowing through the gas until suddenly the gas becomes a good conductor and a spark crosses the gap. He investigated electric breakdown, developed similarity relations governing these processes and measured the emission of light from an electrically excited gas; continuous spectra of rare gases at high pressure were found early in his laboratory by McCallum. During his last years in office, he and Gill developed a theory of the electrodeless discharge in high-frequency electric fields.

Altogether Townsend published probably more than a hundred papers and several treatises: "Electricity in Gases" (1915), "Motion of Electrons in Gases" (based on a lecture which he delivered at the Franklin Institute in Philadelphia in 1925), "Electrons in Gases" (1947), "Electricity and Radio Transmission" (194.3), and "Electromagnetic Waves" (1951).

Townsend was a man of great energy who took much interest in hunting and he had a great sense of humour. He loved talking shop, but still more loved to have an argtument. Looking back at his scientific contributions, one wonders why he was not selected to receive more honours in the field of science. There are many possible reasons. He belonged to the rare class of men who are content and happy with their discoveries and their work. Moreover, his papers were not always easily readable. He seldom attended scientific meetings and took little interest in other people's fields. Although he was reluctant to advertize his or his research men's successes, it is quite remarkable that in spite of that, many of his school have achieved prominence-Moseley, Van de Graaff, Tizard, V. A. Bailey, L. G. H. Huxley, F. Llewellyn Jones and many others.

Townsend was a tireless worker and an excellent talker, but he was sometirnes very absent-minded and stubborn. He once entered a dark room where he found a man struggling with an experiment. $\mathrm{He}$ told him how to perform it, took the readings for him and then left the room without being aware that the man was a candidate in a practical examination. During the First World War, King George V visited the Electrical Laboratory, where cadets of the Signals Corps were trained. At that moment Townsend was writing his magnum opus; he refused to be interrupted in his work, and was not presented.

Townsend was elected to the Royal Society in 1903, and later became a Chevalier de la Légion d'Honneur, a member of the Institute of France and a member of the Franklin Institute, and he was knighted in 1941. He married in 1911 Mary Georgiana, daughter of P. F. Lambert, of Castle Ellen, County Galway, with whom he spent a most happy home life and who survives him with two sons.

A. VON ENGEL

\section{Prof. V. F. Boldyrev}

VASsily F Fedorovich Boldyrev, who died in Moscow on February 25, was born in Saratov on January 20, 1883, educated at the Saratov Gymnasium and the University of Moscow, from which he graduated in 1906, when he joined the staff of the Department of Zoology and Entomology of the Moscow Agricultural Institute (now the Timiriazev Agricultural Academy), at first as an assistant, becoming professor in 1920. The Moscow Institute has been one of the main schools of agricultural entomology in Russia, where many workers in this field received their training, first under the late Prof. N. M. Kulagin, then Boldyrev. This school has always been characterized by giving a much wider general biological education to its students than is usually provided in a course of applied entomology; and Boldyrev has been, above all, an ardent and serious naturalist, able to inspire his pupils with the need to understand the living insects, rather than merely to learn how to kill them.

His own scientific work was extensive and varied. His main original contributions were in the prinstaking studies of courtship behaviour and repro. ductive physiology of Orthoptera. His papers on the subject contain a vast amount of personal observations, described in a lively style rivalling that of Fabre. Other scientific works of Boldyrev dealt with the biology of various insects, while in the field of applied entomology he is deservedly known in the U.S.S.R. as the pioneer of the application of aircraft to the control of insect pests, particularly locusts. Those who, like myself, have been so fortunate as to know Boldyrev personally, will always remember his charming friendliness, deep love of living Nature and keen sense of very kindly humour.

B. P. Uvarov 